\title{
NOTES SUR QUELQUES PLANTES FOURRAGERES DU SAHEL
}

\author{
par M. JOUSSELIN
}

Les pâturages camelins peuvent se classer en trois grandes catégories: Pâturages d'arbres; paturages d'oueds; pâturages de dunes. Cette classification est très imparfaite car de nombreux végétaux herbacés ou arbustifs se rencontrent dans le lit des oueds et sur les dunes.

\section{I. - Pâturages d'arbres.}

Presque tous les arbres ont leurs branches garnies de fortes épines qui ne gênent nullement le dromadaire; celui-ci prend les jeunes branches au milieu de leur longueur, les sectionne ou bien retire sa tête en arrière en arrachant les feuilles; de cette façon les épines se couchent sur la branche.

\section{Mimosées:}

Tala $(\mathbf{A})=\operatorname{Abesar}(\mathrm{A})=\operatorname{Akch}(\mathrm{T})$, dcacia tortilis. - Arbre très répandu dans le Sahel; malgré ses épines, est très recherché du dromadaire qui en consomme en toute saison. Les fruits appelés caroubes ou Tahora en temacheq sont très apprétés.

Tamat (T), Acacia seyal. - Répandu dans tout le Sahel; bon fourrage de qualité identique à l'Acacia tortilis; épines plus petites; gousses plus fines.

Amour (A) = Aggar (T), Acacia arabica. - Se rencontre également dans tout le Sahel mais en moins grande abondance que les précédents; recherché par le nomade pour sa gomme et son écorce qui fournit un excellent tanin; grandes gousses nommées Taggart par les Tem.; peuple les terrains assez compacts.

A'ates (A), Acacia albida. - Bon fourrage, plus fréquent dans l'Azouad.

Irouar (A) = Iouarouar (A), Acacia verek. - Grand arbre du Sahel; grandes caroubes; fréquent dans les terrains sableux.

Sedra bied (A) $=$ Oraf (T), Acacia stenocarpa $\longrightarrow$ Grand arbre plus fréquent dans l'Azouad.

\section{Césalpinées :}

Igargar (A) = Aguerguer (A), Cassia acutifolia. - Peu mangé du bétail, serait purgatif et même toxique.

\section{Burséracèes :}

Adres (A) = Aderas ( $\mathrm{T}$ ), Commiphora africana. - Arbre de moins de 5 mètres; est le premier à reverdir dans l'Azaouad et est consommé à ce moment (septembre à décembre) est ensuite délaissé pour les mimosées. 
M. JOUSSELIN

\section{Chenopodiacées:}

Terchit $(\mathbf{T})=$ Tahara $(\mathrm{T})=$ Askaf $(\mathrm{A})$, Tragänum nudatum. - Arbrisseau de la région de T'aoudéni (Sahara septentrional) apprécié du dromadaire, peu des autres espèces.

\section{Simarubacées:}

Teỉchot $(\mathbf{T})=$ Taborak (T), Balanites aegyptiaca. - Arbre de 4 à 10 mètres; épines vertes; fruits ressemblant à la datte; pâturage accessoire.

\section{Polygonacées:}

Aouarach (T), Catligonum comosum. - Arbre de 2 à 3 mètres de haut; excellent pâturage del'Azouad, pousse dans les lits sablono-limoneux; peu apprécié des autres espèces.

\section{Capparidèes:}

Eiseìn $(\mathbf{A})=$ Aizeur $(\mathrm{A})=$ Tadomet $(\mathrm{T})=$ Tad'hent $(\mathrm{T})$, Boscia senega lensis. $\rightarrow$ Buisson sahélien; 1 à 2 mètres, toujours vert, fleurit pendant l'hivernage.

Atil (A) = Eteil (A) $=$ Adiar (T), Moerua rigida ou crassifolia. - Arbre de 5 aे 10 mètres; fleurit aux premières pluies; reste très longtemps vert; bon fourrage des savanes désertiques, fruits en grappes, sucrés, mais seraient purgatifs.

\section{Papilionacées:}

Irzik (A) = Flemingia faginea = Bouckouk (T.). — Grand arbre assez peu appété du dromadaire qui doit s'y accoutumer avant d'en consommer une certaine quantité; habite les terrains alluvionnaires compacts.

\section{Asclépiadées:}

Asebaïe $(\mathbf{A})=$ Ana $(\mathrm{T})=$ Titarek $(\mathrm{M})$, Leptadenia spartum. - Arbuste pouvant atteindre 3 à 4 mètres; son port rappelle celui du genêt; bon fourrage malgré sa dureté, assez appété, habiteles lits sabloṇneux.

\section{Tiliacées:}

Legleï (A) = Tarakat (T), Grevia betulifolia - Arbre ayant dos feuilles áveo un limbe assez large de couleur jaune verdâtre; le port général de l'arbre rappelle celui du saule; habite les lits sablonneux:

\section{Loranthacées :}

Akaouate (T) = Eklaf (A). Loranthus globiferus. — Il ne s'agit pas d'un arbre mais d'un parasite des mimosées, à la façon du gui, très recherché $d u$ dromadaire, ne-peut pas constituer un pâturage.

\section{II. - Pâturages de dunes.}

\section{Molluginacées :}

Amsrar (A) = Ameterar (T), Gisekia pharnacoïdes. - Bon fourrage quand il 
est vert, plante annuelle rampante, 30 centimètres de long; apparaît après les premières pluies. Se rencontre également dans les oueds.

\section{Zygophyllacées :}

Eglech (A) = Timougloust (T), Tribulus macropterus. - Bon pâturage, vert jusqu'en février dans l'Adrar. Est associé avec le had, pâturage salé.

\section{Cypéracées :}

Taliguit $(\mathrm{T})=$ Telebout $(\mathrm{T})=$ Aligui $(\mathrm{A})$, Cyperus conglomeratus. - Fourrage très grossier, n'est consommé que lorsqu'il est jeune.

\section{Crucifères:}

Ousșit $(\mathrm{T})=$ Akchit $(\mathrm{A})$, g. Farsetia. - Plante annuelle constituant un très bon pâturage; tiges tomenteuses de 20 à 30 centimètres couchées sur le sol, puis redressées à leur extrémité; feuilles allongées; fleurs roses. Pâturage du Nord, se reneontre également dans les lits sablonneux d'oueds.

\section{Papilionacées :}

Amasmaz (A) = Isersan (T), g. Tephrosia. - Pâturage accessoire de dunes, se rencontre également dans certains oueds à lit pierreux; mangé de tous les herbivores.

Téjao (A) = Tachaout (T), Indigofera semitrijuga. - Très bon pâturage, peu abondant dans l'Adrar.

\section{Graminées :}

Serơoum $(\mathrm{A})=$ Taranmout $(\mathrm{T})=$ Enoi du Touat, Aristida acutifolia. Pâturage du nord du Sahel.

Tossouyé (T) = Sbott (A), Aristida pungens. - A l'état sec se nomme Drinn (A) et à l'état vert Illik; herbe vivace de 60 centimètres; feuilles dures identiques à celles du jonc.

\section{Rhamnacées:}

Tabagalt n'édiat (T), Zizyphus lotus. — Jujubier, très accessoire.

\section{III. - Pâturages d'oueds.}

\section{Ficol̈dées :}

Amedressal $(\mathrm{T})=$ Egratin traïb $(\mathrm{A})=$ Aguertin, Trianthema pentendra. Plante annuelle rampante à racine pivotante se présentant en touffes de 15 à 30 centimètres de diamètre; excellent fourrage très appété du dromadaire.

\section{Amaranthacees :}

Tachelanretail $(\mathrm{T})=$ Egratin (A), Amarantus blitum. $\rightarrow$ Très bon fourrage un deş premiers à pousser après les premières pluies. 


\section{Rutacées :}

Taguerouft $(\mathbf{T})=$ Timougloust traïb $(\mathrm{T})=$ Tadressa $(\mathrm{A})$, Tribulus terrestris. Très bon pâturage à l'état vert mais serait sujet à provoquer des météorisations chez le dromadaire venant de faire une cure salée; pousse en tous terrains; apprécié de tous les herbivores.

\section{Acanthaccos:}

Tekaneï (T) = Eflich (A), Blepharis edulis. - Excellent fourrage pouvant atteindre 10 à 25 centimètres, inflorescence en épi quadrangulaire garni d'épincs; flcurs blcues; très rochorchó du dromadaire à l'ćtat vert et même à l'état sec.

\section{Légumineusés:}

Ediat $(\mathbf{A})=$ Taguinguillit $(\mathrm{T})$, Tephrosia polystachia. Légumineuse à fleurs rouges très recherchée du dromadaire, mais dangereuse consommée humide de rosée, car provoque des météorisations. Pousse principalement dans les lits pierreux.

\section{Crucifères :}

Jir-jir (A) = Aelouat (T), Shouvia arabica. - Plante annuelle de 0,50 à 1 mètre de haut, forte tige, larges feuilles. Fourrage très aqueux recherché du dromadaire, doit être associé avec des pâturages secs (arbres). Ne serait mangé de préférence que la nuit? Pousse dans les savanes désertiques rocailleuses, les fissures ensablées de rochers; pâturages du nord de la zone de nomadisation.

Tasselarh $(\mathrm{T})=$ Tabazouaguet $(\mathrm{T})$, Morettia canescens. - Plante vivace rameuse de 30 à 60 centimètres de diamètre, feuilles assez larges. Fourrage de la saison des pluies, abondant danș les dépressions de plaines; excellent fourrage pour tous les herbivores.

\section{Salsolacées:}

Issin (T) = Ressal (A), Salsola foetida. - Excellent fourrage salé de l'Adrar.

\section{Chénopodiacèes:}

Had $(\mathrm{A})=$ Tachara $(\mathrm{T})$, Cornulaca monacantha. - Excellent fourrage salé consommé pendant l'hiver, doit être associé avec d'autres végétaux moins aqueux. Pousse dans les plaines sablonneuses et graveleuses du nord de la zone de nomadisation. Bien mangé du dromadaire mais peu des autres espèces.

\section{Convolvulacées :}

Imsiken (T), Convolvolus fatmensis. - Très bon fourrage d'oued.

\section{Portulacées:}

Alora (A), Portulaca oleracea, pourpier commun $\rightarrow$ Fourrage très accessoire d'oueds et parfois de dunes.

\section{Molluginacées:}

Ameterar (T) = Amsrar (A), Gisekia pharnacö̈des (voir pat. de dunes). 


\section{Graminées:}

Cram-eram, Cenchrus catharticus. - Fourrage d'oueds ot de dunes ne se rencontrant d'après Aug. Chevalier qu'au-dessous du vingt et unième parallèle. Excellent et abondant fourrage de l'A zaouad où il constitue à lui seul des pâturages. Plante annuelle de 30 à 40 centimètres. Selon son état de végétation est appété.

Initi (A) = Ouazzaï ('T) Iorsqu'il est sec.

Gasbah $(\mathrm{A})=$ Akhallas (T) lorsqu'il est vert.

Alémose (T) $=$ Noir (A). - Sous ce vocable sont rangées un grand nombre de Stipćes du g. Lristida; certaines sont consommées par le dromadaire et portent des noms particuliers :

Icardanella (T), Aristida plumosa.

Tessamet (T), Aristida adcientionis. poussent dans les lits sablonneux.

Macoubah (A) = Afazour (T), Panicum turgidum. - Panicée formant de grosses touffes pouvant atteindre 2 mètres, tiges très dures; n'est consommé que lorsqu'il est jeune. Pâturage accessoire abondant dans les lits sablonneux, limoneux et pierreux.

Tougourit (T) = Askanit (A), Latipes senegalensis. - Plante annuelle consommée à la fin de la saison des pluies.

Teloloud (T) = Sag el mohor (A), Aristida stipö̈des. - Plante annuelle de la fin de la saison des pluies; 60 centimètres à $1 \mathrm{~m}$. 20 de haut, assez bon fourrage. 